\title{
OMT: A DYNAMIC AUTHENTICATED DATA STRUCTURE FOR SECURITY KERNELS
}

\author{
Somya D. Mohanty ${ }^{1}$, Mahalingam Ramkumar ${ }^{2}$ and Naresh Adhikari ${ }^{3}$ \\ ${ }^{1}$ Department of Computer Science, University of North Carolina - Greensboro, \\ Greensboro, USA \\ ${ }^{2}$ Department of Computer Science and Engineering, Mississippi State University, \\ Starkville, USA \\ ${ }^{3}$ Department of Computer Science and Engineering, Mississippi State University, \\ Starkville, USA
}

\begin{abstract}
We introduce a family of authenticated data structures - Ordered Merkle Trees (OMT) - and illustrate their utility in security kernels for a wide variety of sub-systems. Specifically, the utility of two types of OMTs: a) the index ordered merkle tree (IOMT) and b) the range ordered merkle tree (ROMT), are investigated for their suitability in security kernels for various sub-systems of Border Gateway Protocol $(B G P)$, the Internet's inter-autonomous system routing infrastructure. We outline simple generic security kernel functions to maintain OMTs, and sub-system specific security kernel functionality for BGP subsystems (like registries, autonomous system owners, and BGP speakers/routers), that take advantage of OMTs.
\end{abstract}

\section{KEYWORDS}

Security Kernels, Broader Gateway Protocol (BGP), Authenticated Data Structure (ADS)

\section{INTRODUCTION}

Any system can be seen as a network of sub-systems, each with a specific role in the operation of the system, interacting with each other according to system-specific and/or role-specific rules. For an ever increasing range of systems, some or all sub-systems take the form of a computer, or a collection of computers (most often a server with one or more back-end servers). For example, sub-systems in the domain name system (DNS) have roles like zone authorities, who create DNS resource records (RR) pertaining to the zone; authoritative name servers, that are chosen by the zone authority to disseminate DNS RRs for the zone; and local (or preferred) name servers, that iteratively query authoritative name servers to resolve queries from clients. Similarly, subsystems in the inter-domain routing infrastructure for the Internet - the Border Gateway Protocol (BGP) - have different roles like autonomous system (AS) owner; AS registry, that assigns AS numbers to AS owners; IP registry that issues (through IP registrars and ISPs) chunks of IP addresses, or IP prefixes (a chunk of consecutive addresses) to AS owners; and BGP speakers for an AS, authorized by the AS owner to originate routes for IP prefixes owned by AS.

Undesired functionality in any hardware/software component of a sub-system may be exploited by an attacker to cause sub-system to misbehave. Undesired functionality may be deliberately hidden malicious functionality (HMF), or accidental bugs. Attackers who exploit undesired functionality may be personnel with legitimate access to the sub-system, or anyone who can take advantage of remotely exploitable HMF/bug to exert some control over the sub-system. For DOI: $10.5121 /$ ijcnc.2016.8401 
example, an attacker can a) compromise a BGP speaker (in a router) to send incorrect routing information; or b) compromise a computer used by the AS administrator to modify the AS policies/preferences; or c) compromise a computer of an administrator in the IP/AS/DNS registry to make duplicate address/AS number assignments.

\subsection{Security Kernel}

It is far from practical to assure the integrity of every hardware/software component in every component of every sub-system. One possible approach to secure systems is to mandate that all important sub-systems should be associated with an appropriate security kernel that vouches for the integrity of (system-specific and role-specific) tasks performed by the sub-system. Specifically, all components of the sub-system are assumed to be untrustworthy; only the security kernel is trusted.

The security kernel for a system/sub-system is also referred to as the trusted computing base (TCB) for the system/sub-system. The TCB for any system is "a small amount of software and hardware that security depends on, and that we distinguish from a much larger amount that can misbehave without affecting security" [1]. For purposes of this paper, the exact nature TCB is not important. For example, the TCB for any sub-system could take the form of a dedicated hardware security module, or a software module executed on a general purpose platform, with some special protections [2] to guarantee that the security kernel will run unmolested, etc.

In the rest of this paper we shall assume that the security kernel for a sub-system is a set of functions executed by a read-proof and write-proof module $T$. It is essential that the security kernel functionality is deliberately constrained to be simple - to permit consummate verification of the functionality, and thereby, rule out the presence of undesired functionality within the security kernel.

Some of the components of the security kernel will necessarily be specific to the nature of the sub-system whose operation is assured by the module - the security kernel functionality for a DNS server will be different from that of an IP registry or a BGP speaker. Nevertheless, to simplify testing of the security kernel functionality, it is advantageous to possess efficient $r e$ usable components of the security kernels, with potential to be useful in a wide range of subsystems. The specific contributions of this paper are: a) an efficient resuable authenticated data structure (ADS), an ordered merkle tree (OMT), and b) illustration of utility of OMTs in a broad range of security kernels (for a broad range of sub-systems).

\subsection{Ordered Merkel Tree}

An ADS $[3,4,5,6,7]$ is a strategy for obtaining a concise cryptographic commitment for a set of records. Often, the commitment is the root of a hash tree. Any record can be verified against the commitment by performing a small number of hash operations. An ordered merkle tree (OMT) is an ADS that is derived as an extension of the better known merkle hash tree. Similar to a plain merkle tree, an OMT permits a resource (computation and storage) limited module to track the records in a dynamic database of any size, maintained by untrusted components of the associated sub-system. Using an OMT (instead of a plain merkle tree) permits the resource limited module to additionally infer a few other "useful holistic properties" regarding the database. For illustrating the broad utility of OMTs, we explore the security kernel functionality necessary for assuring the operation of various BGP sub-systems like IP and autonomous system (AS) registry/registrars, AS owners, and BGP speakers, etc. 
The rest of this paper is organized as follows. In Section 2 we introduce OMTs, and discuss two types of OMTs - the index ordered merkle tree (IOMT) and the range ordered merkle tree (ROMT). In Section 3 we provide an overview of BGP. We enumerate the desired assurances regarding the operation of BGP and suggest high level designs of the security kernel functionality utilizing OMTs to guarantee the desired assurances (to the extent the security kernels are trusted). In Section 5, we suggest other possible applications of OMTs and offer our conclusions.

\section{ORDERED MERKEL TREE}

The merkle hash tree [8] is a data structure constructed using repeated applications of a a preimage resistant hash function $h($ ) (for example, SHA-1). Figure 1 depicts a tree with $N=16$ leaves. In practical merkle tree applications each leaf can be seen as a record belonging to some database.

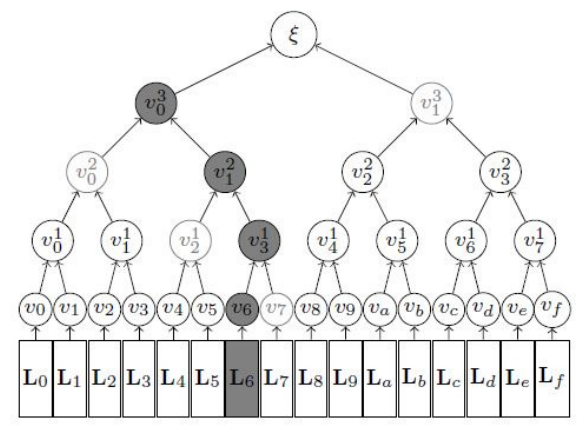

Figure 1. A binary hash tree with 16 leaves. Nodes $v_{6}, v_{3}^{1}, v_{1}^{2}, v_{0}^{3}$ (filled gray) and root $\xi$ are ancestors of

$$
\text { leaf } L_{6} \cdot v_{6}=\left\{v_{7}, v_{2}^{1}, v_{0}^{2}, v_{1}^{3}\right\} \text { are complementary" to } v_{6} \text {. }
$$

A tree with $N$ leaves has a height of $L=\log _{2} N$. At level 0 of the tree are $N$ leaf-nodes, one corresponding to each leaf, typically derived by hashing the leaf. At the next level (level 1) are $N / 2=N / 2^{1}$ nodes, each computed by hashing together a pair of "sibling" nodes in level 0 . Level $i$ has $N / 2^{i}$ nodes computed by hashing a pair of siblings in level $i-1$, and so on, till we end up with a lone node $\xi$ at level $L-$ the root of the binary tree. A tree with $N=2^{L}$ nodes has $2 N-1$ nodes distributed over $L+1$ levels, where $L=\left\lceil\log _{2} N\right\rceil$. Two nodes node $v_{i}^{j}$ and $v_{i+i}^{j}$ at level $j$ are siblings if $i$ is even (else $v_{i-1}^{j}$ and $v_{i}^{j}$ are siblings). Two siblings - the left sibling $u$ and the right sibling $v$ are hashed together to obtain the parent node as $p=h(u, v)$. Given a value $v_{i}^{0}$, the index $i$ of the leaf node, and the set of $k$ complementary nodes, it is trivial to identify the sequence of $k$ hash operations necessary to map a leaf node to the root. We shall represent by

$$
y=f_{m}\left(v_{i}, i, v_{i}\right),
$$

a sequence of $k$ hash operations to obtain the sub-tree root $y$ from a leaf-node with value $v$ and position index $i$. 


\subsection{OMT Leaves and Node}

An ordered merkle tree (OMT) is an extension of the merkle tree with the imposition of a special structure for the leaves of the tree. Every leaf is of the form.

$$
L=\left(A, A^{\prime}, \omega_{A}\right)
$$

Corresponding to a leaf $\left(A, A^{\prime}, \omega_{A}\right)$ is a leaf node computed as

$$
\begin{aligned}
v_{A} & =H_{L}\left(A, A^{\prime}, \omega_{A}\right) \\
& = \begin{cases}0 & A=0, \\
h\left(A, A^{\prime}, \omega_{A}\right) & A \neq 0 .\end{cases}
\end{aligned}
$$

In addition, unlike a plain merkle tree which is intended primarily for dynamic databases with a static number of records (leaves), OMTs are intended to be used for scenarios where leaves may need to be inserted/deleted. For this purpose it is advantageous to redefine the operation of mapping two siblings $u$ and $v$ to their parent $p$ as

$$
p=H_{V}(u, v)= \begin{cases}u & \text { if } v=0 \\ v & \text { if } u=0 \\ h(u, v) & \text { if } u \neq 0, v \neq 0\end{cases}
$$

In other words, the parent of two nodes is the hash of the two child nodes only if both children are non-zero. If any child is zero, the parent is the same as the other child. The parent of $u=v=0$ is $p=0$.

An OMT leaf with the first field set to zero is an empty leaf, represented as $\Phi$. The leaf hash corresponding to an empty leaf is 0 . As introducing an empty leaf node (corresponding to an empty leaf) does not affect any other node of the tree, any number of empty leaves may be seen part of the tree.

\subsection{OMT Types}

OMTs can be seen as falling under two broad categories depending on the interpretation of the first two values. In the first category are index ordered MTs (IOMT), where the first value is interpreted as an index, the second value is the next higher index in the tree. For the leaf corresponding to the highest index the next index is the least index. The third value $\omega_{A}$ in a leaf $\left(A, A^{\prime}, \omega_{A}\right)$ provides some information regarding index $A$. For example, $\omega_{A}$ could be the hash of the contents of a database record with index $A$. It is also possible that $\omega_{A}$ is a root of another OMT, in which case $A$ is an index of a database (which may consist of any number of indexed records).

In an IOMT, existence of a leaf like $(432,562, \omega)$ indicates that no leaf exists for indexes between 432 and 562. A wrapped around leaf like $(796,241, \omega)$ indicates that no leaf exists for indexes greater than 796, and for indexes less that 241 . 
For range ordered MT (ROMT) the values $A$ and $A^{\prime}$ represent the range $\left[A, A^{\prime}\right)$ of some quantity associated with the third value $\omega_{A}$. For example, a leaf like $(432,562, \omega)$ indicates that the quantity $\omega$ is associated with a range $[432,562$ ) (or $432 \leq x<562$ ). For example, an ROMT may be used to represent a look up table (LUT) for some function $y=f(x)$. In such an ROMT each leaf indicates a range of the independent variable $x$, corresponding to which the function evaluates to the dependent variable $y=\omega$ (the third value in the leaf).

\subsection{OMT Properties.}

Some of the important properties of OMTs are as follows. The leaf hash corresponding to an empty leaf $\Phi$ is zero. A tree with root 0 can be seen as a tree with any number of empty leaves. For a tree with a single leaf, the leaf hash is the same as the root of the tree. The existence of a leaf $\left(A, A, \omega_{A}\right)$ in an OMT indicates that the leaf is the only leaf in the tree (in which case the root will be the same as the leaf hash $\left.H_{L}\left(A, A, \omega_{A}\right)\right)$. Existence of a leaf like $\left(1,3, \omega_{1}\right)$ is proof that no leaf exists with first field in-between 1 and 3 . Existence of a leaf like $\left(7,1, \omega_{7}\right)$ is proof that no leaf exists with first field less than 1 and that no leaf exists with first field greater than 7 . As leaves are ordered virtually, the actual physical ordering of leaves has no inherent meaning. Thus, swapping leaves of an OMT does not affect the integrity of the database represented by the OMT.

For both IOMT and ROMT, a leaf with a first field $A$ can be inserted only if a leaf with first two fields that circularly encloses $A$ exists. For inserting a leaf the contents of two leaves in the tree will need to be modified; and empty leaf $\Phi$ will be modified to become the newly inserted leaf, and the second value of the enclosing leaf will need to be modified.

A place-holder is a non-empty leaf whose insertion does not change the interpretation of the database. For an IOMT, a leaf of the form $\left(A, A^{\prime}, 0\right)$ (third value zero) is a place holder. Introduction of a place holder for an index $A$ does not change the database in any way, as both existence of place holder for index $A$ and non-existence of a leaf for index $A$ implies that "no record exists for index $A$." Thus,

$$
\begin{aligned}
& \left(3,4, \omega_{3}\right),\left(1,3, \omega_{1}\right),\left(4,7, \omega_{4}\right),\left(7,1, \omega_{7}\right) \text { and } \\
& \left(3,4, \omega_{3}\right),\left(1,3, \omega_{1}\right),\left(4,5, \omega_{4}\right),(5,7,0),\left(7,1, \omega_{7}\right)
\end{aligned}
$$

which correspond to before and after insertion of a place holder for an index 5 , represent an identical database. For an ROMT, a place holder is a leaf with third value the same as the third value of the enclosing leaf. Specifically, inserting a leaf can be seen as a process of splitting a leaf (for example), $\left(4,7, \omega_{4}\right)$ into two leaves (for example) $\left(4,5, \omega_{4}\right)$ and $\left(5,7, \omega_{4}\right)$. Specifically, both

$$
\begin{aligned}
& (1,3, a),(3,4, b),(4,7, c),(7,1, d) \text { and } \\
& (1,3, a),(3,4, b),(4,5, c),(5,7, c),(7,1, d)
\end{aligned}
$$

represent an identical database. Before insertion, the leaf $(4,7, c)$ indicated that values $4 \leq x<7$ are associated with $c$. Nothing has changed after the range is split into two, as values $(4 \leq x<5)$ and values $(5 \leq x<7)$ are associated with the same quantity $c$. While operations like swapping leaves in any OMT or insertion/deletion of a place holder do not change the contents of the database, they will result in a change in the root of the tree - say from $r$ to $r^{\prime}$. Such roots are considered as equivalent roots. 


\subsection{OMT Functions for Security Kernels}

The module $T$ is assumed to possess limited protected storage, and expose well defined interfaces to the associated untrusted sub-system. Such interfaces can be used by an untrusted sub-system (say) $A$ to demonstrate the integrity of databases stored by the sub-system, and request $T_{A}$ associated with sub-system $A$ to attest verified records.

For attesting records or contents of records (for verification by other sub-systems, or security kernels in other sub-systems) every module is assumed to possess a unique identity, and secrets used for authenticating messages. For example, the secret could be a private component of an asymmetric key pair, which is used for signing messages. In this case, the public key of the module is certified by a trusted key distribution center, attesting the integrity of the module. Alternately one or more secrets could be provided by a trusted key distribution center to each module. Only modules that have been verified for integrity and issued such secrets by the trusted key distribution centers will be able to use their secrets to compute a pairwise secret with other modules attested by the KDCs. Such pairwise secrets may be used to compute message authentication codes for attesting the integrity of the contents of a record.

Apart from secrets provided by trusted KDCs or certified by trusted certificate authorities, every module is assumed to spontaneously generate a random self-secret $\chi$ which is used for authenticating memoranda to itself. For example, after executing (say) $z=f_{m}(x, i, v)$, a module may issue a memoranda to itself to remind itself that it has already verified that " $z$ is an ancestor of $x . "$

As we shall see very soon, the self-memoranda in this scenario is a value $\rho=h(V 1, x, z, \chi)$ computed as a function of the type $V 1$ of the memoranda, the values $x$ and $z$, and the secret $\chi$. No entity other than the module can fake such a memorandum. Thus, if values $x, z$, and $\rho$ are provided as inputs to the module, the module can safely conclude that " $z$ is an ancestor of $x$."

In the rest of this section we provide an algorithmic description of generic OMT functions suitable for security kernels for a wide range of systems/ sub-systems. OMT functions issue different types of self-memoranda. Such self-memoranda may then be used by other systemspecific (or role-specific) security kernel components of the same module. As an illustration of how such memoranda can be used by other system-specific security kernel components of the same module, in a later section we outline the use of such memoranda in security kernels for various BGP sub-systems.

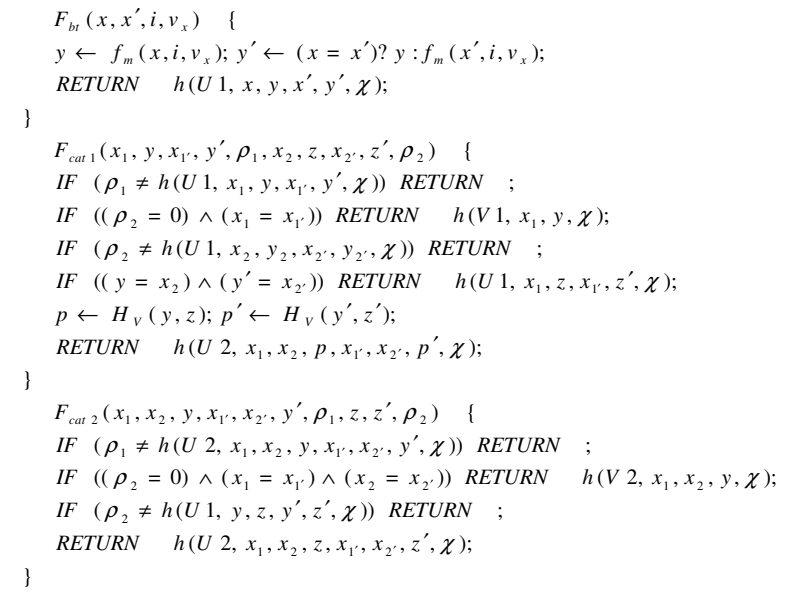

Figure 2. Verification and Update Memoranda. 


\subsection{OMT Memoranda}

Five different types of memoranda are issued by OMT functions.

A certificate of type $U 1$ is issued by functions $F_{b t}()$ and $F_{c a t 1}()$. The inputs to $F_{b t}()$ include a leaf node $x$ in a subtree, the index $i$ of the leaf node (in the sub-tree), and complementary nodes $v$. The root of the subtree can now be computed as $y=f_{m}(x, i, v)$. The function also accepts another value $x^{\prime}$ and computes $y^{\prime}=f_{m}(x, i, v)$ (using the same complementary nodes). The certificate of type $U 1$ issued by this function, viz,

$$
\rho=h\left(U 2, x_{1}, x_{2}, p, x_{1^{\prime}}, x_{2^{\prime}}, p^{\prime}, \chi\right)
$$

states that "(it has been verified by me that) $y$ is the root of a sub-tree with leaf node $x$, and if $x \rightarrow x^{\prime}$ then $y \rightarrow y^{\prime}$." More generally, such a certificate implies that $y$ is an ancestor of $x$, and that if $x \rightarrow x^{\prime}$, then $y \rightarrow y^{\prime}$.

Functions $F_{\text {cat } 1}()$ and $F_{\text {cat } 2}()$ combine self memoranda to issue (in general) more complex selfmemoranda. $F_{c a t 1}()$ accepts inputs necessary to verify the integrity of two type $U 1$ certificates. If the second certificate is 0 , and if in the first certificate binding $x_{1}, y, x_{1^{\prime}}, y$ if $x_{1}=x_{1^{\prime}}$ (implying merely that $y$ is an ancestor of $x_{1}$, a certificate of type $V 1$, viz., $\rho=h\left(V 1, x_{1}, y, \chi\right)$ is issued.

If the child in the second certificate $x_{2}$ is the same as the parent $y$ in the first certificate, the two certificates are combined to issue a single certificate of type $U 1$ binding the child $x_{1}$ in the first certificate to the parent $z$ in the second certificate. Else, $F_{\text {cat } 1}()$ computes $p=H_{V}(y, z)$ and $p^{\prime}=H_{V}\left(y^{\prime}, z^{\prime}\right)$ to issue a certificate of type $U 2$

$$
\rho=h\left(U 2, x_{1}, x_{2}, p, x_{1^{\prime}}, x_{2^{\prime}}, p^{\prime}, \chi\right)
$$

to the effect that that " $x_{1}$ and $x_{2}$ are leaf nodes of a sub-tree with root $p$, and if $x_{1} \rightarrow x_{1}$ ' and $x_{2} \rightarrow x_{2^{\prime}}$ then $p \rightarrow p^{\prime}$. Note that if $y$ is an ancestor of $x_{1}$ and $z$ is an ancestor of $x_{2}$, then $p=H_{V}(y, z)$ is simultaneously an ancestor of $x_{1}$ and $x_{2}$.

Function $F_{\text {cat } 2}()$ extends the common ancestor $y$ of two nodes to an ancestor $z$ of $y$. In other words, $F_{\text {cat } 2}()$ combines a $U 2$ certificate with a $U 1$ certificate to produce a $U 2$ certificate. If only a certificate of type $U 2$ is provided as input to $F_{c a t 2}()$ with $x_{1}=x_{1^{\prime}}$ and $x_{2}=x_{2^{\prime}}$, bound to $y=y^{\prime}, F_{\text {cat } 2}()$ issues a certificate of type $V 2$ binding two nodes $x_{1}$ and $x_{2}$ to a common ancestor $y$.

Certificates of type $U 1$ and $U 2$ are useful for simultaneously verifying and updating the root of the tree. Certificates of type $V 1$ and $V 2$ are useful in scenarios where only verification is required. Functions $F_{p h}(), F_{s w}()$ and $F_{c e}()$ create certificates that bind equivalent roots. A certificate of $\rho=h\left(E I, y, y^{\prime}, \chi\right)$ attests to the equivalence of IOMT roots $y$ and $y^{\prime}$. A certificate $\rho=h\left(E R, y, y^{\prime}, \chi\right)$ attests to the equivalence of ROMT roots $y$ and $y^{\prime}$. 
Through a certificate of type $U 2, F_{s w}()$ recognizes the relationship between two roots resulting from swapping two leaves. As swapping leaves does not affect the integrity of an IOMT or an ROMT, the roots are equivalent for both IOMT and ROMT. Thus, depending on the value $o$ which identifies the type of request ( $o=1$ for ROMT certificate) $F_{s w}($ ) outputs a $E I$ or $E R$ certificate.

Function $F_{p h}()$ issues equivalence certificates binding roots before and after deletion of a place holder. The input $o=1$ is a request to issue a $E R$ certificate (else, the request is for an $E I$ certificate). If no certificate is provided as input to $F_{p h}()$ (or $\rho=0$ ), one root is assumed to the root of an empty tree, and the equivalent root is after insertion of the first place-holder for an index $A$. For both IOMT and ROMT the first place holder will be $(A, A, 0)$, and the root after insertion will be $H_{L}(A, A, 0)$.

If $\rho \neq 0$ this function interprets $\left(A, A^{\prime}, \omega_{A}\right)$ (with leaf hash $\left.x_{1}\right)$ and a place-holder $\left(A^{\prime}, B^{\prime}, \omega\right)$ (with leaf hash $x_{2}$ ) as two leaves in a tree with root $y$. If $o=1$ (ROMT) the place holder has $\omega=\omega_{A}$, else (for an IOMT), $\omega=0$. If the place holder is the first leaf it needs to be modified to $\left(A, B^{\prime}, \omega_{A}\right)$ (leaf-hash $x_{1^{\prime}}$ ) and the second leaf to an empty leaf (leaf hash 0 ). The certificate $\rho$ attests that modifying two leaves $x_{1}$ and $x_{2}$ to $x_{1^{\prime}}$ and $x_{2^{\prime}}$ is equivalent to changing the root from $y$ to $y^{\prime}$. Hence, $y$ and $y^{\prime}$ are equivalent roots.

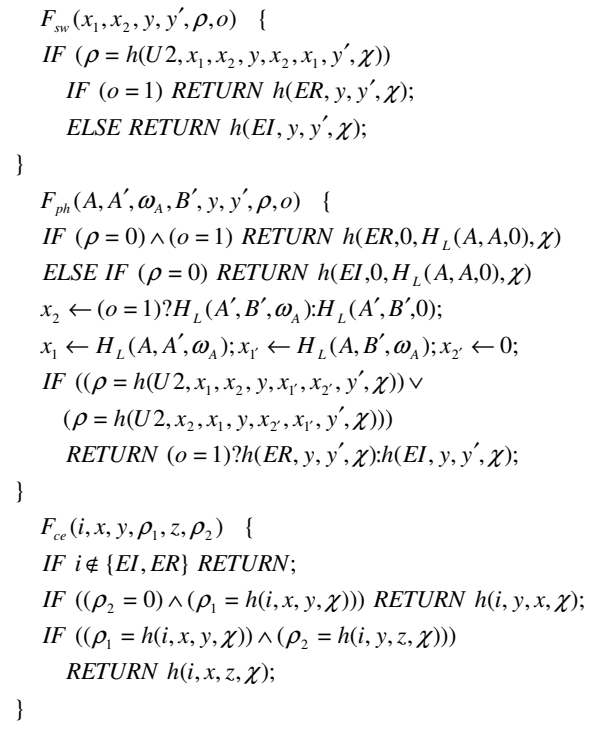

Figure 3. OMT Functions for Issuing Equivalent-Root Memoranda.

\section{BGP SUBSYSTEMS}

The Internet is an interconnection of autonomous systems (AS) [9], [10]. Each AS owns one or more chunks of the IP address space, where the number of addresses in each chunk is a power of 2. IP chunks are represented using the CIDR (classless inter-domain routing) IP prefix notation. For example, the IP prefix 132.5.6.0/25 represents $2^{32-25}$ IP addresses for which the first 25 bits are the same as the address 132.5.6.0, viz., addresses 132.5.6.0 to 132.5.6.127. An AS registry assigns AS numbers to AS owners. AS owners may acquire ownership of IP prefixes from an IP registry (through IP registrars, or ISPs). 
While each AS may follow any protocol for routing IP packets within their AS, all ASes need to follow a uniform protocol for inter-AS routing. The current inter-AS protocol is the border gateway protocol (BGP), where AS owners employ one or more BGP speakers to advertise reachability information for IP prefixes owned by the AS. Specifically, every BGP speaker recognizes a set of neighboring BGP speakers. Neighbors may belong to the same AS or a different AS. The main responsibility of BGP speakers are a) originate BGP update messages for prefixes owned by the AS, and convey such originated messages to neighbors of other ASes; $b$ ) relay BGP update messages received from neighbors to other neighbors; and c) aggregate destination prefixes (that can be aggregated) for reducing the size of routing tables.

BGP is a path vector protocol. BGP update messages communicated between BGP speakers indicate an AS path vector for a prefix. Specifically, a BGP update message

$$
\left[P_{a},(A, B, C, D), W_{d}\right]
$$

from a speaker $S_{d}$ (belonging to the last AS in the path) indicates that prefix $P_{a}$ owned by the first AS $A$ in the path. $W_{d}$ is the weight of the path.

\subsection{BGP Updates}

A BGP speaker may receive multiple paths for the same prefix. All such paths are stored by the BGP speaker in the incoming routing information database (RIDB-IN). However only the best path for a prefix may be copied to the outgoing database (RIDB-OUT), and advertised to other BGP speakers. Most often a BGP speaker is a component of a router which uses entries in RIDBOUT (best path for different prefixes) to forward IP packets.

\subsubsection{BGP Weights}

The best path is the one with the maximum weight. Several parameters are used to compute the weight of a BGP path. For simplicity, in this paper we restrict ourselves to some of the more important weight parameters, i) pre-path weight; ii) local preference iii) AS path length; and iv) multi-exit descriptor (MED).

The pre-path weight is assigned at time of origination. If two paths for the same prefix have the same pre-path weight, then the the local preference is considered (higher the better). If both prepath weight and local preference are the same, the AS path length (number of ASes in the path) is considered. The longer the path, the lower the weight. If the path lengths are also the same, then the MED weight is considered (higher the better).

Local Preference and MED: Every BGP speaker recognizes a set of other BGP speakers as neighbors. Every neighbor is associated with two weight parameters - a local preference, and an MED. From the perspective of a speaker $S_{a}$. That $L_{b}$ is the local preferenc of $S_{b}$ implies that for all paths received from $S_{b}$ the local preference component of the weight should be reset to $L_{b}$. That $M_{b}$ is the MED of $S_{b}$ implies that for all paths advertised to $S_{b}$, the MED component of the weight should be set to $M_{b}$. Local preference and MED weights are assigned only to neighbors that are speakers of foreign ASes. 
Processing Received BGP Updates: When a BGP update message is received from a foreign speaker $S_{b}$ (of AS $B$ ) the steps to be taken by a speaker $S_{a}$ (AS $A$ ) are as follows: 1 ) increment hop-count; 2) add own AS $A$ to the path vector; 3 ) change local preference to value $L_{b}$; 4) set next hop to $S_{b}$; and 5) store path in RIDB-IN. When a path is received from a speaker $S_{a^{\prime}}$ belonging to the same AS, no component of the weight is changed, and the AS number is not inserted.

Relaying and Originating BGP Updates: For relaying a BGP message for a prefix $P$ to a BGP speaker $S_{b}$ in a foreign AS, the steps to be taken by speaker $S_{a}$ are: a) among all paths for the same prefix, choose the path with the highest weight; b) change the MED component of weight to; c) advertise the path with modified weight. For originating a path (for owned prefixes), the pre-path weight is set, and the MED is set to that of the foreign neighbor. Such originated paths are not sent to speakers of the same AS (as paths to IP addresses within the AS are established using an intra-AS protocol). For relaying a BGP update message (for a prefix owned by a foreign AS) to a speaker $S_{a^{\prime}}$ of the same AS, simply choose the path with the highest weight and send it without changing the weight.

Policies and Preferences: The choice of BGP speakers for the AS, the prefixes for which a speaker may originate BGP update messages (along with their pre-path weights), neighbors of each speaker, along with their local preference and MED weights, etc., can be seen as policies and preferences specified by the AS owner to influence the weights assigned to BGP paths.

Aggregation: One of the major benefits of CIDR prefixes come from the fact that BGP speakers may aggregate prefixes. If two consecutive prefixes $A$ and $B$ (say 126.5.4.0/25 and 126.5.4.128/25) and can be aggregated into a single prefix $C$ (126.5.4.0/24) if the next hop for prefixes $A$ and $B$ is the same. The speaker that performed the aggregation is the originator for the aggregated prefix.

\section{SECURITY KERNELS FOR BGP SUB-SYSTEMS}

Thus far we have outlined generic security kernel functionality for issuing OMT certificates. In this section we consider other sub-system specific security kernel functionality for various BGP sub-systems like AS and IP registries, AS owners, and BGP speakers.

For simplicity, we shall assume a single registry for both AS numbers and IP addresses. All security kernel modules have a unique identity. Let $U$ be the identity of the module associated with the registry. One module is associated with every AS owner. We shall assume the identities of an AS owner modules to be the same as the AS number. Each BGP speaker is associated with a module. We shall assume that the identity of BGP speaker modules to be the IP address of the router/BGP speaker. We also assume the existence of module functionality for authentication/verification of messages exchanged between modules. Specifically, we shall represent such functionality as

$$
\mu=f_{a}\left(X, Y,\left\{v_{1}, v_{2}, \ldots\right\}\right) \text { and }\{0,1\}=f_{v}\left(X, Y,\left\{v_{1}, v_{2}, \ldots\right\}, \mu\right)
$$


the process of authentication (by module $X$, using $f_{a}()$ ) and verification (by module $Y$, using $\left.f_{v}()\right)$ of a message conveying values $\left\{v_{1}, v_{2}, \ldots\right\}$, from module $X$ to module $Y$. Function $f_{a}()$ outputs a authentication code $\mu$. Function $f_{v}()$ outputs a binary value (TRUE if authentication $\mu$ is consistent, or FALSE).

The identity $U$ of the registry module is known to all AS owner modules. The registry module $U$ delegates AS numbers and IP prefixes to AS owner modules. AS owner modules will only accept delegations from $U$. AS owner modules in turn delegate IP address ranges they own to one or more BGP speaker modules.

Some of the specific desired assurances regarding the operation of BGP are as follows:

1. AS number can not have more than one owner; an IP address can not be owned by one or more ASes. Such assurances should be guaranteed even if the computers employed by the registry have been compromised by an attacker.

2. AS owners can only delegate address ranges owned by the AS to BGP speakers.

3. Notwithstanding the possibility that a router/ BGP speaker may be under the control of an attacker, the following assurances are desired

a) The BGP speaker will only be able to create BGP update messages for prefixes delegated by the AS owner

b) No BGP update message can be created by violating any of the policies / preferences specified by the AS owner (neighboring speakers, local preference and MED, pre-path weights) or BGP rules (only the path with the best weight can be advertised).

c) A speaker will not accept paths which already includes its own AS (to ensure that routing loops can not be created).

d) All BGP speakers will increment the hop count exactly by one.

e) A speaker will be able to aggregate only prefixes for which the next hop is the same speaker.

\subsection{OMTs Used by BGP Subsystems}

The registry and AS owners maintain an ROMT where each leaf indicates a range of IP addresses, and the third value is the AS number (of the AS that owns the address range).

BGP speakers maintain one ROMT, multiple IOMTs, and a plain Merkle tree. A plain Merkle tree is used to maintain a neighbour table with a static number of records. More specifically, for scenarios involving dynamic databases where records can not be inserted or deleted (the dynamics come only from modification of records) OMT is an over-kill; a plain Merkle tree is adequate. The ROMT is used maintaining address ranges for which the speaker can originate BGP updates (owned prefixes and aggregated prefixes).

An IOMT is used for maintaining the RIDB-IN database. More specifically a nested IOMT is used where the root corresponds to a tree with leaves whose indexes are IP prefixes. Corresponding to each prefix the value (third field) is the hash of two IOMT roots. The root of the "path tree" has one leaf for every path for the prefix. The root of the "weight tree" represents the weights of different paths, and enables the module to readily identify the path with the highest weight. The index of leaves in path tree is a function of a quantity that is itself the root of an IOMT. Specifically, the "path vector" IOMT with root has a leaf corresponding to every AS in the AS path. Representing the AS path in this way makes it possible for the module to recognize that it is already in the path, and thereby prohibit creation of routing loops. 


\subsection{Registry Module $U$ and AS Owner Modules}

The registry module maintains an ROMT root $\xi_{r}$, where each leaf indicates ranges of IP addresses, and the AS number of the owner. Unassigned IP chunks have a leaf with value 0 .

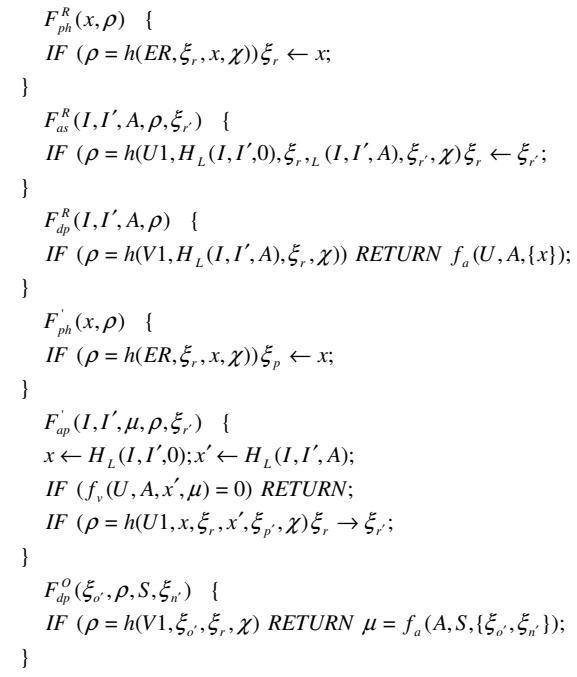

Figure 4. Security Kernel Functionality in Registry and AS Owner Modules.

The function $F_{p h}^{R}()$ can be utilized to insert/delete any place holders in the ROMT by providing a memoranda of type $E R$. The registry employs the function $F_{a s}^{R}()$ to convert the third value of any leaf from 0 to a non zero value. A leaf $\left(I, I^{\prime}, A\right)$ in the ROMT indicates that the IP addresses in the range $I$ and $I^{\prime}-1$ have been assigned to AS $A$. The leaf $\left(I, I^{\prime}, A\right)$ can be conveyed to an AS owner module $A$ using interface $F_{d p}^{R}()$.

AS owner modules also maintain an ROMT with root $\xi_{r}$. The leaves indicate IP addresses owned by the AS. In the tree maintained by the owner of AS $A$ who (for example) owns two non consecutive chunks with addresses between $\left[a, a^{\prime}\right)$ and $\left[b, b^{\prime}\right)$ the ROMT leaves will be $\left(a, a^{\prime}, A\right),\left(a^{\prime}, b, 0\right),\left(b, b^{\prime}, A\right)$ and $\left(b^{\prime}, a, 0\right)$. The function $F_{p h}^{o}()$ can be used to insert/delete place-holders in the tree. Once a place older $\left(a, a^{\prime}, 0\right)$ exists, a delegation $\left(a, a^{\prime}, A\right)$ from the registry module $U$ can be used to update the place holder to a leaf $\left(a, a^{\prime}, A\right)$. Any node in the tree with root $\xi_{r}$ can now be sub-delegated to a BGP speaker. Depending on which prefixes need to be delegated to which BGP speaker the owner can use $F_{p h}^{O}()$ to subdivide owned prefixes and swap positions of prefix leaves, and choose the root of a subtree which includes all prefixes to be delegated to the speaker. Apart from delegating IP prefixes, the AS owner also specifies various preferences as leaves of a hash tree (with root $\xi_{n^{\prime}}$ ). The types of records in this tree include

1) Pre-path weight; a record of the form $[P, o]$ for each owned prefix $P$ that can be originated by the speaker, indicating the pre-path weight $o$.

2) Neighbor preferences record for each neighbor. A record for neighbor $F$ is of the form 
International Journal of Computer Networks \& Communications (IJCNC) Vol.8, No.4, July 2016

$$
N_{F}=\left[F, s_{f}=0, t_{f}=0, A_{f}, L_{f}, M_{f}, \tau_{f}\right]
$$

where $A_{f}$ is the AS number of the neighbor, $L_{f}$ and $M_{f}$ are the local preference and MED weights, and $\tau_{f}$ is the maximum permitted duration between HELLO messages from the neighbor $N$. The values $s_{f}$ and $t_{f}$ are set to zero by the AS owner. Such fields can be modified only by the module of a BGP speaker initialized using the value $\xi_{n^{\prime}}$. The value $s_{f}$ is the time at which a link to $F$ was established. Value $t_{f}$ is the time at which the $F$ was last heard-from.

\subsection{BGP Speakers}

The security kernel of BGP speakers maintains 3 dynamic roots (see Figure 5): the root $\xi_{o}$ of an ROMT is initialized to a value $\xi_{o^{\prime}}$ communicated by the AS owner module; the root $\xi_{n}$ of a Merkle tree (with a leaf corresponding to every neighbour, and a static leaf for every owned prefix corresponding to which BGP speaker can originate BGP updates) is initialized to the value $\xi_{n^{\prime}}$ conveyed by the AS owner module; the root $\xi_{d}$ of an IOMT indexed by IP prefix - the RIDB tree, which is initialized to zero. BGP speakers also maintain a static value $A$ - initialized to the AS number represented by the speaker. During regular operation of the BGP speaker the RIDB root $\xi_{d}$ is updated whenever a BGP update message is received, or if a path is removed (for example) due to loss of link to neighbor.

The neighbor/preferences tree root $\xi_{n}$ is updated whenever a neighbor state is updated. Specifically, corresponding to each neighbor are two dynamic values: a connection identifier $s$ (which is the time at which the connection was initiated) and a time-stamp $t$ (time of last activity in the connection).

The leaves of the ROMT are IP address ranges for which the speaker can originate BGP updates. Originated updates can be for owned IP address ranges or for aggregated prefixes. When initialized, the ROMT root $\xi_{o}$ is a commitment to leaves corresponding to owned IP ranges (delegated by the AS owner module by conveying a root of a sub-tree from its tree of owned prefixes). In all such leaves the third value $a$ is the AS number. The ROMT root $\xi_{o}$ may also be updated for purposes of aggregating CIDR prefixes. Specifically, for any prefix in the RIDB tree the address range and the next hop in the best path to the prefix can be added to the ROMT. Thus, for leaves corresponding to foreign IP ranges the third value is the next hop. Two adjacent prefixes with the same next hop can now be aggregated. More specifically, aggregation corresponds to removing a place-holder. For example, two leaves $\left(I_{1}, I_{2}, x\right)$ and $\left(I_{2}, I_{3}, x\right)$ where $\left[I_{1}, I_{2}\right)$ and $\left[I_{2}, I_{3}\right)$ are two ranges with the same next hop $x$, can be converted to a single leaf $\left(I_{1}, I_{3}, x\right)$ through an equivalence operation.

From the perspective of the BGP speaker modules, corresponding to a BGP update message from a speaker (with IP address) $X$ to a speaker $Y$ is an authenticated message from module $X$ to module $Y$ computed as: 


$$
\left.\mu=f_{a}\left(X, Y,\left\{P, \alpha, l, w_{p p}, w_{l p}, w_{m e d}\right]\right\}\right)
$$

where $P$ is the prefix for which the path is advertised, $\alpha$ is a one-way function of the AS path, $l$ is the path length, $w_{p p}, w_{l p}$ and $w_{\text {med }}$ are respectively the pre-path weight, local preference, MED. The four weights are used to construct a weight represented as

$$
W=\left[w_{p p} w_{l p} M A X-l w_{\text {med }}\right]
$$

Thus, for any prefix the path with the highest weight $W$ is the best path.

Security kernel functions $F_{\text {rel }}^{S}()$ and $F_{\text {orig }}^{S}()$ are used to create such BGP update messages, and $F_{\text {upd }}^{S}()$ is used to process such messages from neighboring speakers and update the RIDB root.

More specifically, $F_{\text {orig }}^{S}()$ is used to originate BGP updates (for own prefixes and aggregated prefixes). Specifically, a path for a prefix $P$ (represented in the origin tree as a leaf with range $\left[I_{1}, I_{2}\right)$ and third value $v$ ) can be advertised only if $a$ ) the third value $v$ is its own AS number, and a leaf exists in the tree with root $\xi_{n}$ for the prefix $P$, conveying the pre-path weight $w_{p p}$ for prefix $P$; or $b$ ) the third value $v$ corresponds to a neighbor with a live link, and no leaf with prefix $P$ exists in the RIDB tree. $F_{r e l}^{S}()$ is used to relay stored BGP paths in the RIDB to neighbors. $F_{\text {rel }}^{S}()$ identifies the best path for a prefix, and only the best path may be advertised. Alternately, information regarding the best path can also be added to the origination tree to aggregate a prefix.

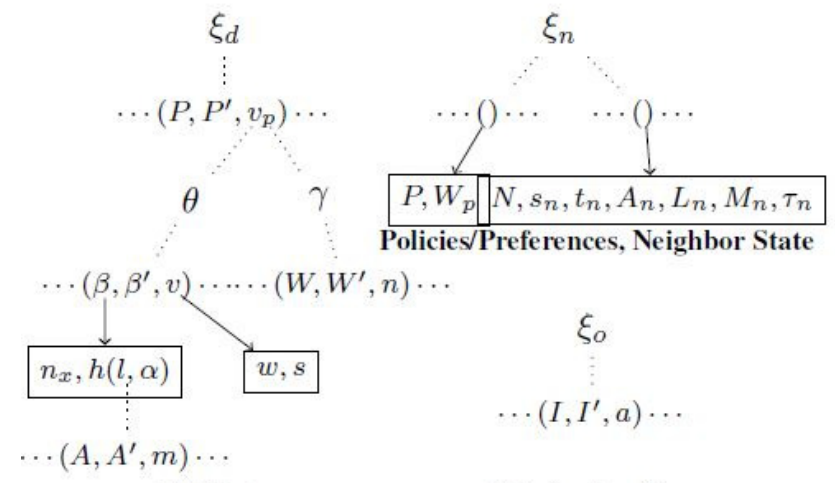

RIDB tree Origination Tree

Figure 5: OMTs Used by BGP Speakers.

Neighbouring BGP speakers maintain a TCP connection over which BGP update messages are exchanged. To keep the connection alive, and for testing the existence of the link, special HELLO messages are exchanged periodically. From the perspective of the security kernel in a speaker $S$ the link to a neighbour $F$ is associated with the link establishment time $s_{f}$ and a timestamp $t_{f}$. 
Once a link has been established, the module $F$ in is expected to confirm their continued presence by periodically sending authenticated time-stamped messages for updating the timestamp $t_{f}$.

In the RIDB-IN, multiple paths, each with possibly different weights, may exist for each prefix. To enable the security kernel to readily determine the path with the highest weight, the plurality of weights for each prefix are maintained as an ordered list. In the weight IOMT, the index of a leaf is a weight, and the value (third field) is the number of occurrences of the weight in the list.

For example, corresponding to a list with four weights $(21,21,34,42)$, three leaves $(21,34,2),(34,42,1),(42,21,1)$ will exist in the weight tree (index 21 occurs twice as indicated by the value field). As in any IOMT, insertion of a place-holder (say for index 5, which signifies "zero occurrences of value 5 in the list)" does not modify the list.

Within the RIDB IOMT a special IOMT is also used to represent AS paths. In the AS path IOMT the the index of leaves are ASes. A tree corresponding to a path of length 5 will have 5 leaves. The value field (third field) is the position in the path. As an example, corresponding to a path $A \rightarrow D \rightarrow B \rightarrow E$ the leaves of the tree will be $(A, B, 1),(B, D, 3),(D, E, 2)$ and $(E, A, 4)$ (note that the value for index $D$ is 2 as $D$ is the second AS in the path).nIn the RIDB IOMT the index of leaves are IP prefixes. The value field in the IOMT is a one way function of two IOMT roots 1) OMT root $\gamma-$ is the root of a weight-IOMT; and 2)IOMT root $\theta$ - the root of an IOMT whose leaves like $\left(\beta, \beta^{\prime}, v\right)$ characterize each path to the prefix.In the IOMT with root $\theta$ the index of leaves are functions of the path; more specifically, in the index $\beta=h(G, h(l, \alpha))$, $G$ is the next hop, $l$ is the path length, and $\alpha$ is the root of an AS-path IOMT root. The value $v$ corresponding to an index $\beta$ is a function of two values - the weight $W$ of the path, and the connection identifier of the next hop that provided the path. If the connection identifier in a path is not the same as the identifier in the neighbor record for that neighbor, then the path is considered as stale (and the weight is set to 0 ).

\subsection{Using Security Kernel Functions in BGP Speaker Module}

BGP speaker modules expose a function $F_{\text {init }}^{S}()$ which is invoked to initialize the module. In the rest of this paper we shall investigate the functionality of a speaker $S$ belonging to an AS $A$. An authenticated message from AS module $A$ (created by using function $F_{d p}^{A}()$ in Figure 6) is necessary for initializing the roots of the neighbor tree to $\xi_{n^{\prime}}$, and the origin tree to $\xi_{o^{\prime}}$.

Any place holder can be added to the IOMT with root $\xi_{r}$ or the ROMT with root $\xi_{p}$. Using function $F_{p h}^{S}()$. Any place holder can also be added to the path tree or weight tree corresponding to any prefix. This can be accomplished using function $F_{p h 2}^{S}()$ which issues a equivalence memoranda of type $E 2$ identifying two roots corresponding to before and after insertion of a place holder in a tree with root $\theta$, or a tree with root $\gamma$, or both. 
Function $F_{S}^{\text {hlo }}()$ can be invoked to create authenticated messages that can be sent to other speakers. This function ensures that speaker $S$ can only connect to speakers explicitly authorized by the AS owner (by providing the initial root $\xi_{n}$ ). Such authenticated messages can be used to create a connection (with a new value of $s$ deemed sufficiently close to the current time $t$ ), and for updating time stamps of neighbors.

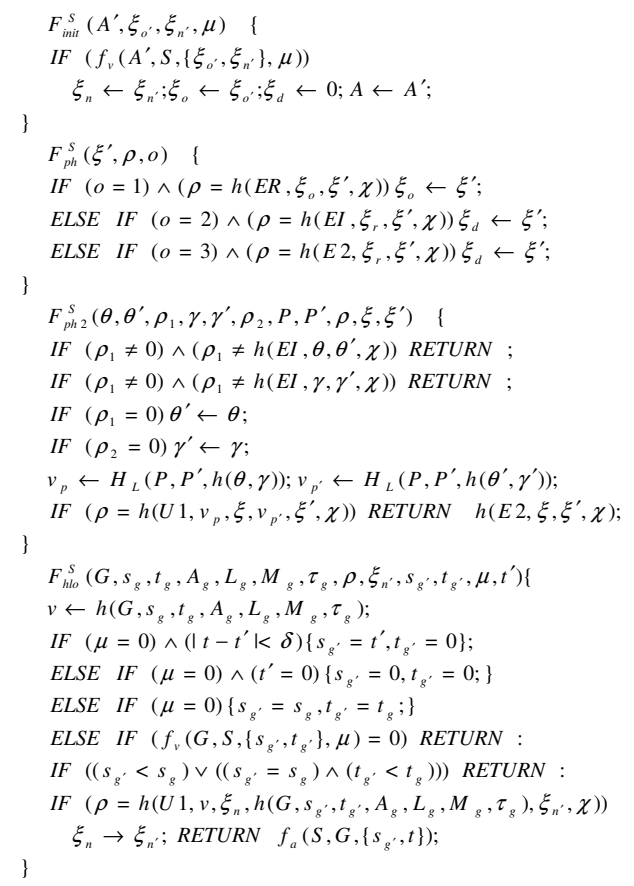

Figure 6: Security Kernel Functions for BGP Speakers.

\subsection{Processing BGP Updates}

Function $F_{\text {upd }}^{S}()$ is invoked to update the RIDB-IN tree - either due to a BGP update message received from a neighbor, or due to loss of link to the next hop. From the perspective of the security kernel the link to the next hop is broken if the time-stamp in the neighbor record is stale. If the current neighbor session identity is different from the session identity of the next hop in the stored path, then the path is assumed to be invalid (as the path was provided during an earlier session). If the neighbor is no longer active, or if the path is invalid, the path weight will be set to 0 .

$F_{\text {upd }}^{S}()$ is invoked to update a path for a prefix $P$. Recall that a prefix $P$ is associated with a path tree root $\theta$ and a weight tree root $\gamma$. A path in the path tree is uniquely identified as a function of the AS-path $\alpha$, path-length $l$, and next hop $N$ : the index of the path is $\beta=h(N, h(l, \alpha))$. The path is associated with a path weight $W_{c}$ and the session identity $s_{n}$ of the next hop. 


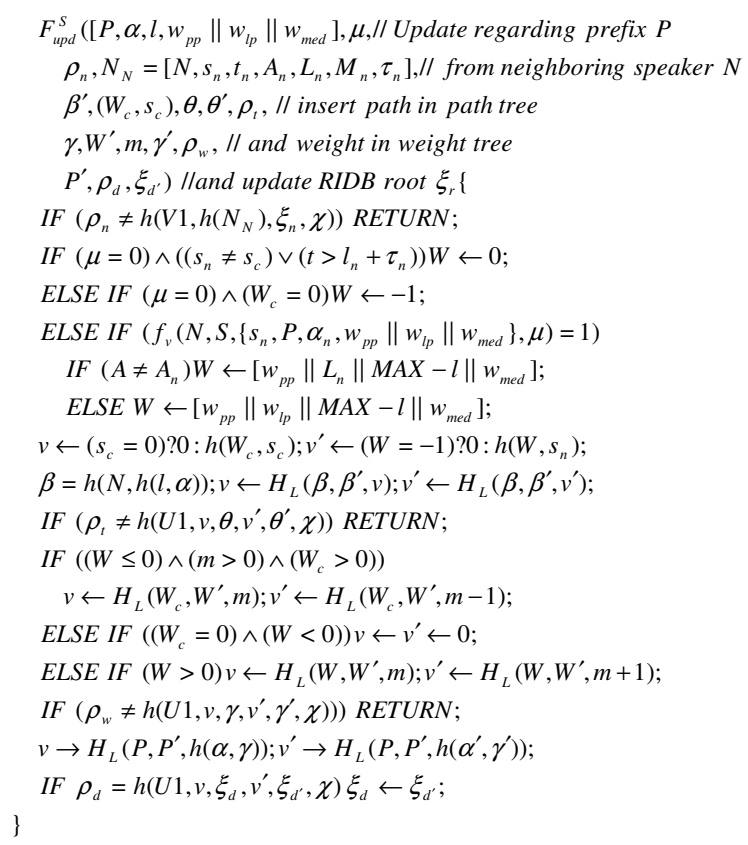

Figure 7. BGP Speaker Security Kernel Functionality for Accepting BGP Updates

Updating the path implies modifying the current weight $W_{c}$ associated with the index $\beta$ to a weight $W$. In addition, modification of the weight requires the weight tree to be modified. Specifically, a) if $W_{c}=0$ and $W \neq 0$ (inserting a path), then the value $W$ has to be added to the IOMT with root $\gamma$; b) if $W=0$ and $W_{c} \neq 0$ (setting path weight to 0 ) then the value $W_{c}$ has to be removed from the tree with root $\gamma$.c $)$ if $W=W_{c}=0$, then $F_{\text {upd }}^{S}()$ is invoked to delete a path with zero weight. In this case no change is necessary to the weight tree root $\gamma$.

For inserting a path $F_{\text {upd }}^{S}()$ is invoked by submitting a received BGP update from a neighbor $N$ specifying path vector $\alpha$, path length $l$, and weights $w_{p p}\left\|w_{l p}\right\| w_{\text {med }}$. The weight for the inserted path is then

$$
W=w_{p p}\|x\| M A X-l \| w_{\text {med }}
$$

where $x=L_{n}$ or $x=w_{l p}$. Specifically, if the neighbor $N$ providing the update belongs to from a foreign AS, the $x=L_{n}$ (the local preference of $X$ ); if $N$ belongs to the same AS, the local preference $w_{l p}$ advertised by $N$ is retained.

For setting weight to zero $F_{\text {upd }}^{S}()$ may be invoked without a BGP message, or a BGP message that withdraws a previously advertised path. A withdraw message from a neighbor indicates $w_{p p}=w_{l p}=w_{\text {med }}=0$. 
In general, updating a path with index $\beta$ (for prefix $P$ ) will require modification to the path tree root $\theta$ and weight tree root $\gamma$ (in the leaf for prefix $P$ ). For incorporating the change in values $\alpha$ and $\gamma$ associated with leaf index $P$, the RIDB root $\xi_{d}$ will need to be modified.

The inputs to $F_{\text {upd }}^{S}()$ include a) a neighbor record $N_{N}$ for $N$ and a $V 1$ memoranda $\rho_{n}$ to verify the integrity of the record against the root $\xi_{n}$ b) $U 1$ memoranda $\rho_{t}$, necessary to update a leaf with index $\beta$ in a tree with root $\theta$, c) $U 1$ memoranda $\rho_{w}$, necessary to increment the counter in leaf with index $W$ (when a path with weight $W$ is inserted), or decrement the counter in a leaf with index $W_{c}$ (when the current weight $W_{c}$ of the path is reset to 0 ), in the weight tree with root $\gamma$, d) $U 1$ memoranda $\rho_{d}$, necessary to update the RIDB-IN root $\xi_{d}$ due to the changes to values $\gamma$ and $\theta$ associated with index $P$; and e) a received authenticated BGP update message $\left[\alpha, l, w_{p p}\left\|w_{l p}\right\| w_{\text {med }}, \mu\right]$ from neighbor $N$.

\subsection{Advertising BGP Paths}

Function $F_{a d v}^{S}()$ is invoked to identify the best path for a prefix and a) advertise the best path (create BGP update) to a neighbor, or b) add the prefix for the path (along with the next hop and session identity of the next hop) to the origination (This is to enable aggregation of prefixes, i.e. two adjacent prefixes with the same next hop and session identity can be aggregated by removing a place-holder in the ROMT) tree. $F_{a d v}^{S}()$ can also be invoked to create a BGP update to withdraw a path with weight 0 .

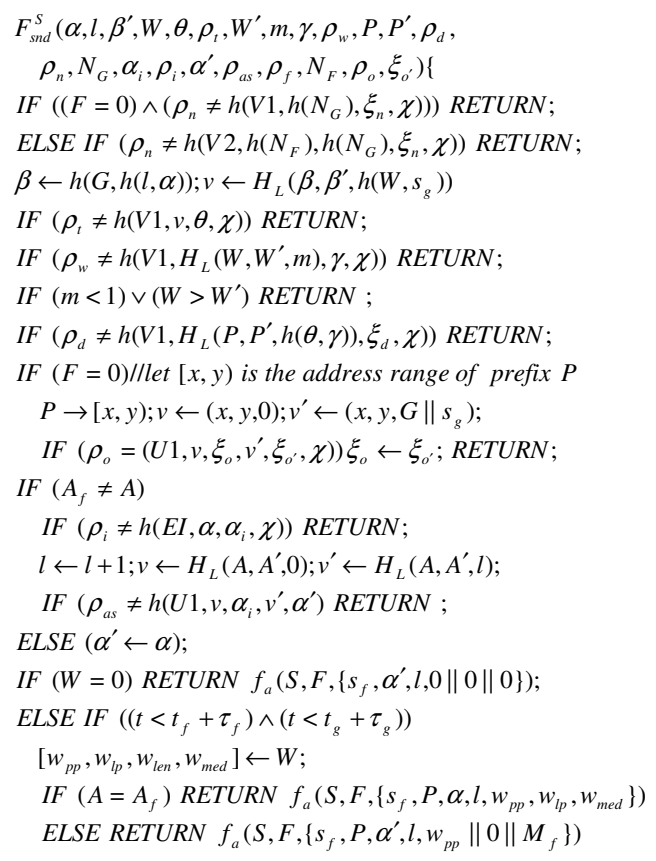

Figure 8. BGP Speaker Security Kernel Functionality for Relaying BGP Updates. 
If $W$ is the best weight for prefix $P$ then a leaf $\left(W, W^{\prime}, m\right)$ with $W^{\prime}<W$ and $m \neq 0$ should exist in the tree with root $\gamma$. This is demonstrated using a memorandum of type $V 1$. There should also exist a leaf for an index $\beta=h(G, h(l, \alpha))$ in the tree with root $\theta$ associated with values $s_{g}$ and $W$. For this purpose a $V 1$ memoranda is necessary to demonstrate the integrity of a neighbor record for $G$ against $\xi_{n}$, and another $V 1$ memoranda is required to demonstrate the integrity of the leaf with index $\beta$ against $\operatorname{root} \theta$. Finally, another $V 1$ memoranda is required to demonstrate the integrity of values $\theta$ and $\gamma$ associated with index $P$ against the RIDB root $\xi_{d}$. Now that the best path (described by next hop $G$, AS vector $\alpha$, path length $l$ and weight $W$ ) has been identified,

1. a leaf with range $[x, y)$ corresponding to prefix $P$ can be added to the origination tree indicating next hop and session identity $G \| s_{g}$, or

2. a BGP update for prefix $P$ can be created and sent to a neighbor $F$.

In the former case, updating the origination tree will require a leaf $(x, y, 0)$ to be modified to $\left(x, y, G \| \mathrm{P} s_{g}\right)$ where $P \equiv[x, y)$. For updating the leaf of the origination tree, a $U 1$ memoranda is required as input to $F_{a d v}^{S}()$.

Before a BGP message for a path can be advertised to a foreign neighbor $F$, the path vector and path length have to be modified (to insert own AS number). If the path vector root is currently $\alpha$, and the length is currently $l$, the value $l$ should be incremented, and a new leaf needs to be inserted into the IOMT with root $\alpha$. Specifically, the new leaf will have index $A$ (AS number of the speaker) and value $l+1$. More specifically, a place holder for $A$ needs to be inserted in a tree with root $\alpha$, following which the place holder can be updated to modify the third field from 0 to $l+1$. Thus, a memoranda of type $E I$ (for inserting a place holder) and a memoranda of type $U 1$ (for updating the place-holder) are required as inputs.

\subsection{Originating BGP Updates}

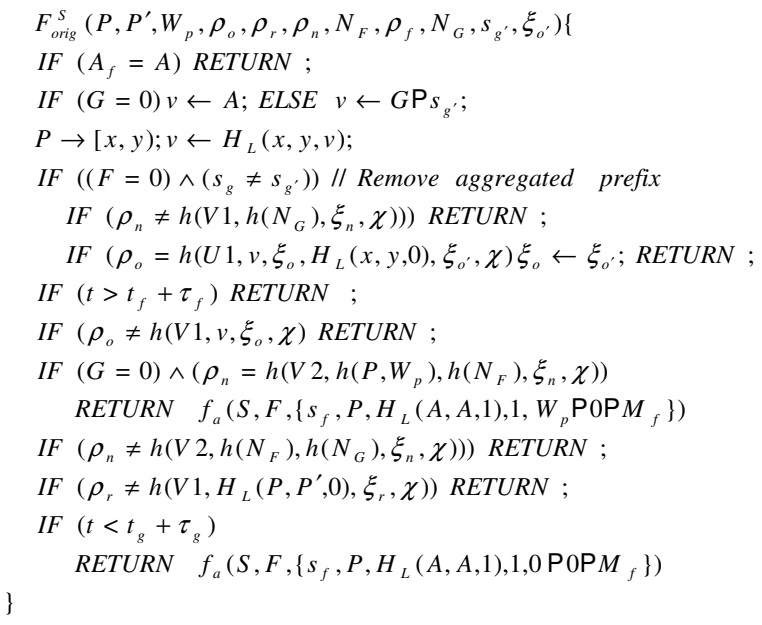

Figure 9. BGP Speaker Security Kernel Functionality for Originating BGP Updates. 
$F_{\text {orig }}^{S}()$ is used to advertise path information for two categories of prefixes 1) prefixes owned by the AS; and 2) aggregated prefixes. Specifically, in leaves corresponding to owned prefixes in the origination tree, the third value will be its own AS number $A$. Corresponding to other leaves the third value will be a neighboring speaker $G$ (next hop for the prefix) and a session identity $s_{g^{\prime}}$ of $G$ (at the time the prefix was added to the origination tree.) An owned range $[x, y)$ can be converted into a prefix $P$ and advertised to a neighbor $F$ only if a record $\left(P, w_{p p}\right)$ exists in the neighbor/policies tree with root $\xi_{n}$. A certificate of type $V 2$ is provided as input to simultaneously verify the integrity of the neighbor record $N_{F}$ and record $\left(P, w_{p p}\right)$ in the neighbor tree. To advertise an aggregated prefix $P$ a $V 1$ memoranda attesting the integrity of the next hop neighbor record $N_{G}$ is required. In addition, a $V 1$ certificate is required to demonstrate that prefix $P$ does not exist in the RIDB-IN tree. If the next hop $F$ (to whom the origination message is to be sent) is set to $F=0$, then $F_{\text {orig }}^{S}()$ interprets this as a request to delete an aggregated leaf for prefix $P$ with third value $G \| s_{g^{\prime}}$. To remove the aggregated prefix the third value $G \| s_{g^{\prime}}$ is set to 0 . For this purpose a certificate $\rho_{o}$ of type $U 1$ is required as input.

When a BGP message is originated for an owned prefix or an aggregated prefix the MED weight is set to to value $M_{f}$ (for the intended receiver $F$ ) provided by the AS owner; the local preference is set to 0 ; for owned prefixes the pre-path weight is set to the value $W_{p}$ prescribed by the AS owner, and for aggregated prefixes the pre-path-weight is set to 0 .

\section{RELATED WORK ANd CONCLUSIONS}

In a large majority of security-kernel based approaches in the literature, the purpose of the security kernel is to ensure that verified software is executed unmolested on an untrusted platform. In the trusted computing group (TCG) approach based on the trusted platform modules (TPM) only the security kernel is trusted to realize the assurance that that "only pre-verified software can take control of the platform."

The security kernel, or the TCB for the TCG-TPM approach, can be seen as composed of three roots of trust - the root of trust for storage (RTS), reporting (RTR) and measurement (RTM). The RTS and RTR are offered by a hardware TPM bound to an untrusted platform. The RTM includes "all essential hardware required to run software." Most often, the "essential hardware" includes the CPU, RAM, CPU-RAM bridge and BIOS.

The purpose of the RTM is to measure every unit of software that takes control of the CPU. The unit of software is typically a file, and the measure is the file hash. The trusted BIOS includes software that measures itself, reports the measurement to the TPM, load the next level of software (usually the boot-loader), measure the boot-loader, and report the measurement to the TPM.

If the boot loader can be verified to be free of malicious code then the boot loader loads the next level of code (the operating system kernel), measures the kernel and reports the measurement to the TPM. Similarly the operating system can load other higher level components and report measurements to the TPM. 
The RTS is trusted to securely store measurements; the RTR is trusted to report measurements. Any entity interacting with the untrusted platform can now request the TPM to report the measurements, and may choose to abandon the interaction if the reported measurements differ from expected measurements. This strategy of building a chain of trust starting with the BIOS is the AEGIS model [11] adopted in the TCG approach.

The main issues with the TCG-TPM approach are three fold:

1. Ensuring that software can run unmolested is very little comfort when the software itself becomes too complex to be thoroughly verified. Furthermore, hidden malicious functionality in complex software may actually load other software without reporting their measurements (or reporting arbitrary measurements) to TPM.

2. Lack of a secure binding between the RTM (trusted components of the untrusted platform) and the TPM (which houses RTS and RTR). The implication of this is that the TPM can uncoupled from the RTM, and supplied expected measurements (while the platform runs arbitrary software).

3. The "minimal hardware trusted to run software" may also include peripherals with direct access to RAM. This results in the well known TOCTOU problem [12] in the TCG approach.

In the proposed approach the goal of the security kernel is not to ensure the integrity of the all software related to a system/ sub-system. Rather, the goal is to ensure only some very specific sub-system specific properties. For example, if the TCG approach is used to secure the AS/IP Registry, every computer used by the Registry should be TPM enabled, and every piece of software that can take control of any computer should be carefully examined to be free of malicious code. However, in the proposed approach, only the simple security kernel functionality outlined in Figure 4 needs to be assured to be clear of undesired functionality.

Most commonly used hash tree based ADSs include the well-known merkle tree [8], skip-lists [7], red-black trees [5], and B-trees [3, 13]. All such ADSs (except the plain Merkle tree) essentially provide the capability to order values in a set (based on some index). The main difference between OMTs and other ADSes like skip-list, red-black trees and B-trees are:

1. In the OMT, the ordering is virtual (the first two fields in an OMT can be seen as a circular link list). In other trees the ordering is physical.

2. An OMT without the third field is functionally equivalent to other trees. The third value in an OMT binds the first value to a record (in an IOMT) or a range to some "owner" (in an ROMT).

Alternately, a skip-list and a merkle tree are together functionally equivalent to an OMT. From this perspective, the main advantage of the OMT is that with a simple tweak to the merkle tree, the OMT realizes the advantages of ordering values (viz., ability to readily determine existence and non-existence of records, maximum/minimum values, etc.) without using an additional tree.

While there is very little algorithmic difference between an ROMT and an IOMT, there is a substantial difference in their functional utility. In this paper we illustrated the utility of an ROMT for registry and AS owner security kernels to maintain database of IP address ranges and the ownership of the range. In a BGP speaker the ROMT additionally enables the speaker to aggregate IP prefixes. The IOMT is used for a wide range of purposes like maintaining the RIDB, AS path trees (one for every path), and weight trees (one for every prefix). 
The current approach to secure BGP is based on the Secure BGP [14] protocol proposed by Kent et.al. This approach employs public key certificates to authenticate communication between ASes (BGP updates) and delegation of AS numbers/IP prefixes. More specifically, a dual certificate system (supported in the back-end by a public key infrastructure (PKI)) is used where the one certificate binds the public key of the AS owner to the operating address space (IP prefix) and AS number, and a second certificate binds routers to an AS. Apart from such static certificates, dynamic certificates are also created by BGP speakers along with every update message. Specifically, such certificates created by every AS in the path seeks to assure the integrity of the AS path vector. Whenever a router receives an update message, it verifies the dual certificates to ascertain the validity of the message. In order to advertise the received message it extends the path by adding itself to the path and signing it (along with the nested signatures of the previous hops) with its own public key. To prevent deletion attacks a speaker in AS A sending an update message to a speaker in AS $B$ also includes the next hop in the signature.

While S-BGP approach is successful in its claims for identity verification (AS owner, routers) and update message integrity, it fails to provide any assurances for the overall operation of a subsystem in the protocol. For example, there are no assurances provided by the protocol guaranteeing that a router will indeed select the best path and that it will strictly abide by the policies and preferences prescribed by the AS owner. The security features of S-BGP protocol does not extend to aggregated prefixes as it is impractical to create static certificates to validate "ownership" of aggregated prefixes. This is a severe disadvantage of S-BGP as much of the advantages of CIDR stem from the ability to aggregate prefixes.

In the proposed approach the simple security kernel associated with BGP speakers ensure that the speakers can only advertise the best path, that all preferences and policies of the As owner will be strictly adhered to. More importantly, the assurances also extend to aggregated prefixes.

\section{REFERENCES}

[1] B. Lampson, M. Abadi, M. Burrows, and E. Wobber, "Authentication in distributed systems: Theory and practice,” ACM Transactions on Computer Systems, vol. 10, pp. 265-310, 1992.

[2] E. R. Sparks, "A Security Assessment of Trusted Platform Modules Computer Science Technical Report," Power, pp. 1-29, 2007

[3] P. T. Devanbu, M. Gertz, C. U. Martel, and S. G. Stubblebine, "Authentic Third-party Data Publication," in Proceedings of the IFIP TC11/ WG11.3 Fourteenth Annual Working Conference on Database Security: Data and Application Security, Development and Directions. Deventer, The Netherlands, The Netherlands: Kluwer, B.V., 2001, pp. 101-112. [Online]. Available: http://dl.acm.org/citation.cfm?id=646118.758638.

[4] A. Buldas, P. Laud, and H. Lipmaa, "Accountable Certificate Management Using Undeniable Attestations," in Proceedings of the 7th ACM conference on Computer and communications security, ser. CCS '00. New York, NY, USA: ACM, 2000, pp. 9-17. [Online]. Available: http://doi.acm.org/10.1145/352600.352604

[5] A. Anagnostopoulos, M. T. Goodrich, and R. Tamassia, "Persistent Authenticated Dictionaries and Their Applications," in Proceedings of the 4th International Conference on Information Security, ser. ISC '01. London, UK, UK: Springer-Verlag, 2001, pp. 379-393. [Online]. Available: http://dl.acm.org/citation.cfm?id=648025.744371

[6] C. Martel, G. Nuckolls, M. Gertz, P. Devanbu, A. Kwong, and S. G. Stubblebine, "A General Model for Authentic Data Publication," Algorithmica, 2004

[7] M. Goodrich, R. Tamassia, and A. Schwerin. Implementation of an Authenticated Dictionary with Skip Lists and Commutative Hashing. In DARPA Information Survivability Conference Exposition II, 2001. DISCEX '01. Proceedings, volume 2, pages 68 -82 vol.2, 2001.

[8] R. C. Merkle, "Protocols for Public Key Cryptosystems," Security and Privacy, IEEE Symposium on, p. 122, $1980 . \quad$ [Online]. Available: http://www.computer.org/portal/web/csdl/doi/10.1109/SP.1980.10006 
[9] Y. Rekhter and T. Li, “A border gateway protocol 4 (bgp-4),” 1995.

[10] Y. Rekhter and P. Gross, "Application of the border gateway protocol in the internet," 1995.

[11] W. A. Arbaugh, D. J. Farbert, and J. M. Smith, "A Secure and Reliable Bootstrap Architecture," in IN PROCEEDINGS OF THE 1997 IEEE SYMPOSIUM ON SECURITY AND PRIVACY. IEEE Computer Society, 1997, pp. 65-71

[12] S. Bratus, E. Sparks, and S. W. Smith, “TOCTOU, Traps, and Trusted Computing," in In Trust 08: Proceedings of the 1st International Conference on Trusted Computing and Trust in Information Technologies, 2008, pp. 14-32.

[13] M.T. Goodrich, R.Tamassia, N. Triandopoulous, and R. Cohen, "Authenticated Data Structures for Graph and Geometric Searching," in Proceedings of the 2003 RSA conference on The cryptographers' track, ser. CT-RSA'03. Berlin, Heidelberg: Springer-Verlag, 2004, pp. 295-313. [Online]. Available: http://dl.acm.org/citation.cfm?id=1767011.1767042

[14] S. Kent, C. Lynn, and K. Seo, "Secure border gateway protocol (s-bgp)," Selected Areas in Communications, IEEE Journal on, vol. 18, no. 4, pp. 582-592, 2000

\section{AUTHORS}

Dr. Somya D. Mohanty is an Assistant Professor at the Department of Computer Science at University of North Carolina - Greensboro. He received his Master's degree in Computer Science from Florida State University and his doctorate from the department of Computer Science and Engineering at Mississippi State University. His doctoral research focuses on designing security kernels for distributed applications. Somya has worked as the Data Scientist/Systems Architect on the Social Media Tracking and Analysis System (SMTAS) project with the

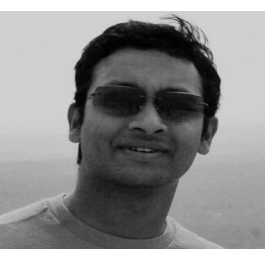
Innovative Data Laboratory at the Social Science Research Center. In the research effort, he designed system architectures capable of handling Big Data and develops algorithms to gain insights from the data in real-time. He also contributed to the server architecture design with the use of dynamic scalable components capable of handling large data influx (Big Data). Somya's other research interests include information/network security, cryptographic protocols, content analysis, machine learning and distributed storage architectures.

Dr. Mahalingam Ramkumar is an Associate Professor of Computer Science and Engineering at MSU. He received his Bachelors degree in Electrical Engineering from University of Madras, India, MS in Electrical Engineering from Indian Institute of Science, Bangalore, India, and $\mathrm{PhD}$ in Electrical Engineering from New Jersey Institute of Technology, Newark, NJ, in Jan 2000. He served as the Chief Technology Officer for a technology start-up in Newark between Feb 2000 to Sep 2002, and as a

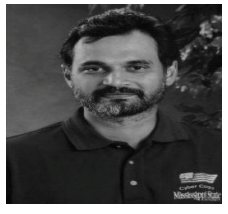

Research Assistant Professor in Polytechnic University, Brooklyn, NY from Oct 2002 to July 2003. His current research interests include trustworthy computing, applied cryptography, and network security. His has authored 2 books, 20 Journal articles/book chapters, and over 70 refereed conference publications.

Mr. Naresh Adhikari is a graduate research assistant in Computer Science and Engineering at MSU. He received his Bachelors degree in Software Engineering from Pokhara University, Nepal and is pursuing $\mathrm{PhD}$ in computer science in MSU under advisorship of Dr. Ramkumar. His current research interests include event detection in high speed network and machine learning.

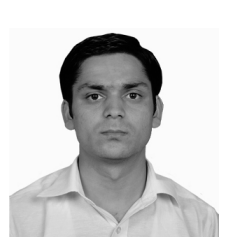

